\title{
Development of a method for suspended sedi- ment transport monitoring by means of ADCP measurements
}

\author{
Rui Aleixo ${ }^{1, *}$, Massimo Guerrero ${ }^{1}$, Nils Ruther $^{2}$, and Siri Stokseth ${ }^{3}$ \\ ${ }^{1}$ University of Bologna, DICAM, via Umberto Terracini 28, 40131 Bologna, Italia \\ ${ }^{2}$ NTNU, Department of Civil and Environmental Engineering, S.P. Andersensveg 5, 7022 Trondheim, \\ Norway \\ ${ }^{3}$ Statkraft AS, Lilleakerveien 6, Postboks 200 Lilleaker, 0216 Oslo, Norway
}

\begin{abstract}
Monitoring stations in rivers and water courses are an important mean to obtain critical data about the different variables that play a role in the hydrodynamics and ecological processes. Measuring suspended sediment concentration often requires the displacement of equipment and manpower to the field. This is often expensive and not practical, in particular during severe weather and flow conditions. A method to determine the suspended sediment concentration as a result of ADCP remote measurements is here presented. This method relies on the relationship between the attenuation to backscatter ratio and the normalized attenuation coefficient. To test this method, data from a field monitoring station in Kokel, on the banks of the Devoll river in Albania, is used.
\end{abstract}

\section{Introduction}

Monitoring stations in rivers and water courses are an important mean to obtain relevant data about the different variables that play a role in hydrodynamics and ecological processes. One of these variables is the suspended sediment transport. Knowing how much sediment is transported in suspension can be useful for example, to estimate the time-life span of a dam's reservoir.

Suspended sediment transport measurements are usually carried by taking samples of water from the river. Depending on the analysis method available it is possible to determine both the concentration and particle size distribution of the sediments. Although relatively simple, this method usually requires human intervention, the programming of an experimental campaign and the displacement of means and technicians to the field. This is not a practical method, in particular during severe weather and flow conditions. Furthermore, the measurement of suspended sediment transport in real time is not possible, and other ways of measuring the suspended sediment transport must be investigated. Recently, some methods to relate sound attenuation with the suspended sediment transport have been presented. An acoustic-based method to determine the suspended sediment concentration as a function of the received echo intensity was introduced by Guerrero et al. [1,2] and it is here presented with an application to a field case study.

\footnotetext{
*e-mail: rui.aleixo@unibo.it
} 


\subsection{Mathematical model}

Using the sonar equation it is possible to find a proxy to determine the suspended sediment concentration (SSC). The sonar equation can be written as [3]:

$$
I_{d B}=C+10 \log \left(k_{s}^{2} M_{s}\right)-20 \log (r \psi)-40\left(\alpha_{w}+\alpha_{s}\right) r \log (e)
$$

Where $I_{d B}$ is the intensity in $\mathrm{dB}, C$ accounts for the instrument parameters, $10 \log \left(k_{s}^{2} M_{s}\right)$ is referred as the backscatter strength and $k_{s}^{2}$ and $M_{s}$ are the backscattering coefficient and the sediment concentration (in $\mathrm{kgm}^{-3}$ ) respectively; $r \psi$ is the geometrical beam spreading and $\psi$ is the near field correction $\left(\psi=1\right.$, in the far-field). $\alpha_{w}$ and $\alpha_{s}$ are the water viscosity and suspended sediment attenuation coefficients, respectively. Equation (1) can be rewritten by grouping in the left-hand side the variables related with the suspended sediment concentration, namely concentration and particle size distribution (PSD) and in the right-hand side the known or measured variables: the received sound intensity, the acoustic beam spreading and the sound attenuation in clear water as modelled according to [4]:

$$
10 \log \left(k_{s}^{2} M_{s}\right)-40 r \alpha_{s} \log (e)=I_{d B}-C+20 \log (r \psi)+40 r \alpha_{w} \log (e)
$$

Different relationships exist for the backscattering strength, and for the two additive mechanisms producing the attenuation. The suspended sediment attenuation coefficient can be written in terms of the normalized scatter and viscous attenuation coefficients, $\zeta_{s s}$ and $\zeta_{s v}$ respectively, as:

$$
\alpha_{s}=\left(\zeta_{s s}+\zeta_{s v}\right) M_{s}=\zeta_{s} M_{s}
$$

Where $\alpha_{s}$ is expressed in Np.m ${ }^{-1}$. The scatter attenuation, $\zeta_{s s}$, is responsible for the energy spreading of the incident beam, whereas the viscous dissipation, $\zeta_{s v}$, is due to the friction produced by particles to fluid relative motions [5-7]. The normalized coefficients $\zeta_{s s}$ and $\zeta_{s v}$ depend on the sound frequency and on type of sediments and should be determined for each site by means of specific and eventually periodic calibrations with in-situ sampling collection. Their sum is referred as normalized attenuation coefficient and represented as $\zeta_{s}$.

\subsection{The Attenuation to backscatter ratio (ABR) at single frequency}

Figure 1 depicts some examples of echo profiles, from which the suspended sediment concentration can be determined. Echo profiles A and B illustrate a case of progressive attenuation. Echo profile $\mathrm{C}$ depicts the case of a strong attenuation and finally profile $\mathrm{D}$ a case where the attenuation is only visible after about $3 \mathrm{~m}$. In case of echo profiles A and D it is possible to identify the right bank position by the peak in the echo profile. The data measured inside the right banks has therefore no physical meaning. The left-hand side of equation (2) includes the terms that are related with the backscatter $\left(k_{s}^{2} M_{s}\right)$ and the attenuation term $\left(\alpha_{s}\right)$. Their difference, as stated by equation (2) is only a function of the instrument constants, the measured echo intensity and the water attenuation coefficient.

This difference can be expressed as a ratio:

$$
A B R=\frac{\zeta_{s} M_{s}}{10^{\log \left(k_{s}^{2} M_{s}\right)}}=\frac{\zeta_{s}}{k_{s}^{2}}
$$

In particular, it is worth noting that the attenuation to backscatter ratio at a given frequency depends on the actual PSD features (e.g., diameter (d), standard-deviation (std) and skewness), as first pointed out by Guerrero and Di Federico [1]. This means that a limited 


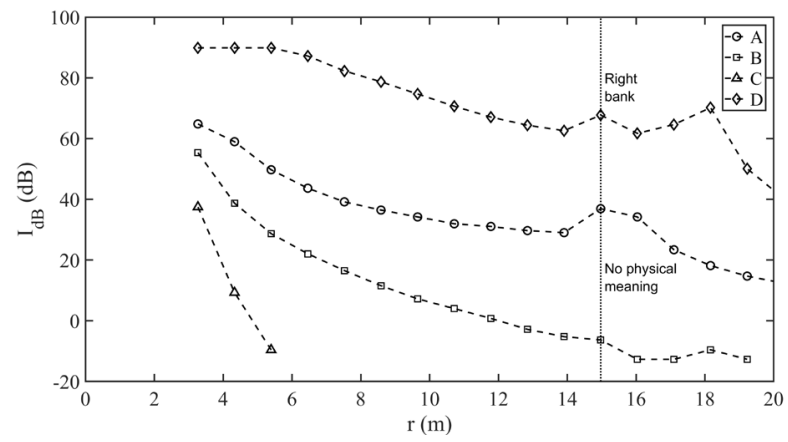

Figure 1. Example of echo profiles (in $\mathrm{dB}$ ) with the indication of the right bank. Profile A measured on 2016/02/24 at 2:59:12, Profile B measured on 2016/02/11 at 5:05:21, Profile C measured on 2016/02/11 at 00:02:21 and Profile D measured on 2016/11/05 at 23:54:34.

region for d-std values may be associated to a known attenuation to backscatter ratio at a given frequency (Figures $2 \mathrm{a}$ and b). Also, as pointed out by Guerrero et al. [2] the normalized coefficients $\left(\zeta_{s s}\right.$ and $\zeta_{s v}$ ) are also function of the PSD features. By measuring the ABR it is possible to identify a range of d-std values, that may be used to estimate the normalized attenuation coefficients.

Figure 2 (a) depicts the attenuation to backscatter ratio as a function of the diameter (with standard deviation as a parameter) and Figure 2 (b) depicts the attenuation to backscatter ratio as a function of the standard deviation using the diameter as parameter for two different frequencies, one the double of the other [1].

From Figures 2 (a) and (b) it is possible to identify different behaviours: with increasing sound frequency, the curves are parallelly shifted towards smaller values of the ABR, illustrating the increasing weight of the backscattering strength over the attenuation processes. On the other hand, a lower frequency increases the weight of attenuation compared to backscatter that result in smaller flattening of the ABR curves.
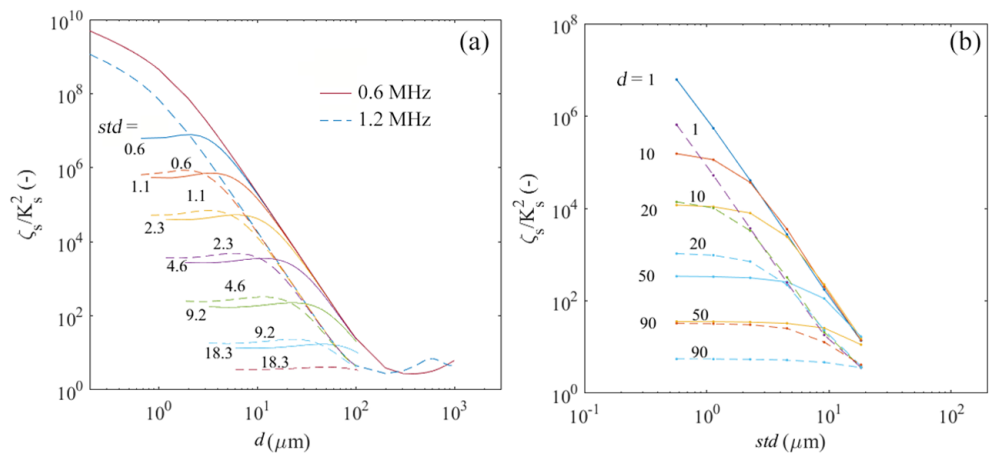

Figure 2. Attenuation to backscatter ratio $\left(\zeta_{s} / k_{s}^{2}\right)$ change with mean size and standard deviation for two well-spaced frequency and the same PSDs set. 


\subsection{Suspended sediment concentration determination}

Hanes [7] discussed the possibility of measuring sand and clay concentrations in uniform suspensions by means of single-frequency acoustic measurements. Moore et al. [5] showed that suspended sediment concentration measurements during floods could be made using horizontal aligned beams.

To determine the concentration a simplified version of equation (1), valid for homogeneous sediment content of suspended sediments, is used. For this case, the change of backscatter strength is negligible along the ranging distance, resulting that the sediment concentration is a function of the measured echo profile slope, $d I_{d B} / d r$, by means of the attenuation coefficient, $\alpha_{s}$ :

$$
\zeta_{s} M_{s}=-\alpha_{w}-\frac{1}{2 r}-\frac{1}{40 \log e} \frac{d I_{d B}}{d r}
$$

The sediment concentration is therefore given by:

$$
M_{s}=\left(-\alpha_{w}-\frac{1}{2 r}-\frac{1}{40 \log e} \frac{d I_{d B}}{d r}\right) \frac{1}{\zeta_{s}}
$$

The normalized attenuation coefficient $\zeta_{s}$ has therefore to be determined for the specific conditions of the place where the measurement has to be carried. One way to estimate $\zeta_{s}$ is by means of the ABR. As stated in section 1.2, $\zeta_{s}$ can be related to ABR through the actual $\mathrm{PSD}$. The ABR and $\zeta_{s}$ dependence from the independent variables consisting in PSD features at a given frequency are here used to obtain the abacus of Figure 3. This abacus depicts a set of PSDs, identified by a pair of values $\mathrm{d}$ and std, in terms of ABR and $\zeta_{s}$ values at $0.6 \mathrm{MHz}$.

Determining the ABR value, the attenuation is determined by projecting the ABR value in the vertical axis by means of the corresponding d-std function. The obtained value of $\zeta_{s}$ is then used in equation (6) to determine the concentration.

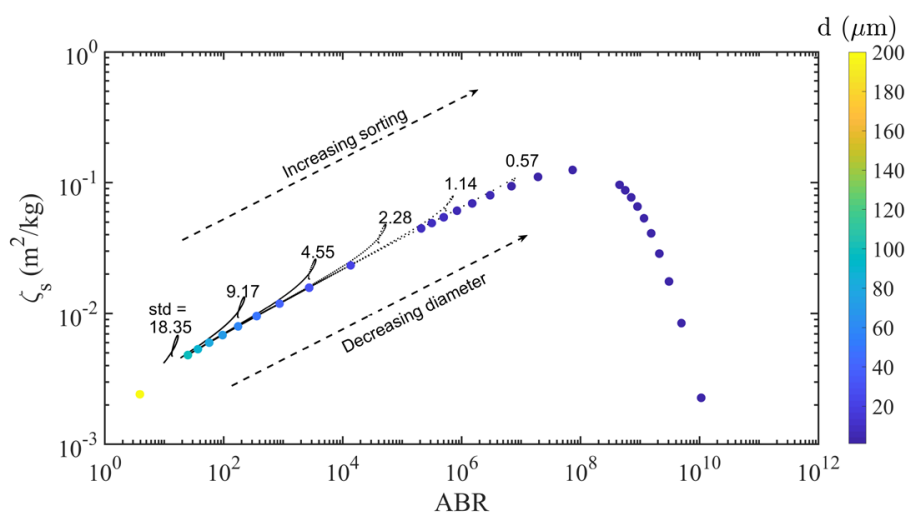

Figure 3. The conversion function for a monogranular sediment distribution and to some PSDs to convert from attenuation to backscatter ratio (ABR) to concentration. Drawn family of curves determined for Skewness $=8,1 \mu \mathrm{m} \leq \mathrm{d} \leq 200 \mu \mathrm{m}$ and standard deviations indicated on the figure. 


\section{Experimental setup}

The Kokel measuring station is located in the left bank of the Devoll river, near Kokel, Gramsh city, Albania. This monitoring station has been continuously operating since 2016. It has proven to be quite sturdy having resisted to some severe floods events and requiring only minor maintenance periods.

The Devoll is a mountain stream flowing in $1 \mathrm{~km}$ wide alluvial valley with typical braid morphology. The water discharge ranges between $10^{1} \mathrm{~m}^{3} \mathrm{~s}^{-1}$ and $10^{3} \mathrm{~m}^{3} \mathrm{~s}^{-1}$ (order of magnitude). In Kokel, a passage between steep sides keeps the entire flow within $30 \mathrm{~m}$ width section (Figure 4) that was suitable to monitor the flow discharge. Flow velocity measurements carried at this section have shown $10 \mathrm{~ms}^{-1}$ during floods.

The measuring station consists of several instruments namely: two side looking ADCPs working at two different frequencies $(0.6 \mathrm{MHz}$ and $1.2 \mathrm{MHz})$. These ADCPs allow for the simultaneous measurement of the flow velocity profile and of the echo profiles along the same cross-section. Temperature and pressure sensors are integrated in the ADCPs, allowing for the measurement of both water temperature and level. Integrated in the measurement station is also an ISCO sediment sampler, and a pressure transducer in the river bed. The instruments are remotely accessed via GSM network and measured data are transmitted to a server using the same network. This makes it possible to have both instruments and data available $24 \mathrm{~h}$ a day, 365 days a year.

Figure 4 depicts the Kokel measuring station and the Devoll river section profile at the measuring station.
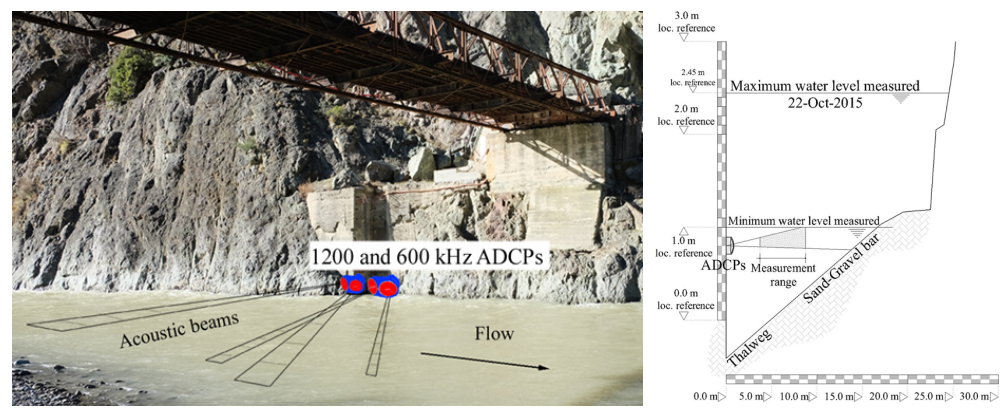

Figure 4. Kokel measuring station with the indication of the ADCPs location and profile of the river section at the measuring station location.

\section{Data processing and results}

ADCP data is acquired every 2 minutes. A box-average with 30 values is applied to obtain hour-averages of the measured data (both velocity and echo profiles).

For the echo analysis leading to the sediment concentration other operations are made, namely: the determination of the near-field and the instrument constant $C$. The near-field is assessed using the sensor's area, $A_{o}$ and the sound wavelength, $\lambda$ as $r_{n f}=A / \lambda$. To compute the parameter $C$ values different approaches can be used, namely, based on a direct measurement of $M_{s}$ in a calibration wise approach, and another one, based on the dispersion of the backscatter values distribution. For the present case results from one measurement of sediment properties in the Devoll river were used and the obtained valued was $C=179.4 \mathrm{~dB}$. It is important to stress here, that one value measured on a single day, may not be sufficient for an accurate analysis of a long time-series. 


\subsection{Assessing $\zeta_{s}$ value for concentration vs. attenuation}

If there is not any information regarding the transported sediments PSD, one possibility to determine the $\zeta_{s}$ is to use the monogranular function as described in Figure 3, using the linear part $\left(10^{1}<\mathrm{ABR}<10^{8}\right)$.

Some water samples obtained from the Devoll river, at the measuring station, were analysed by means of optical diffraction techniques in order to determine the sediments PSD. The obtained data is plotted in Figure 5 that defines the conversion function for lower ABR values.

The obtained validation is valid for a restrict range of ABR values (Figure 5). This is in contrast with the set of resulting relationships including monogranular PSDs and given standard deviations in Figure 3. These results show the need for more detailed sampling campaigns, made in different flow conditions (e.g. flood events) to obtain a wider range for the required relationship between $\zeta_{s}$ and ABR.

From the behaviour of the family of curves defined by d, std and skewness, it is possible to see that, for increasing values of ABR (small diameters), the different PSDs curves tend to the monogranular case (Figure 5). It is therefore reasonable to assume that the monogranular case is a limiting value for lower $\zeta_{s}$ (higher values of concentration). For the the non-validated domain of ABR, to obtain the limit case for higher values of $\zeta_{s}$ (smaller values of concentration), the maxima of each curve is taken to obtain a linear relationship as depicted in Figure 5.

These two curves are then used to convert the calculated ABR values into $\zeta_{s}$.

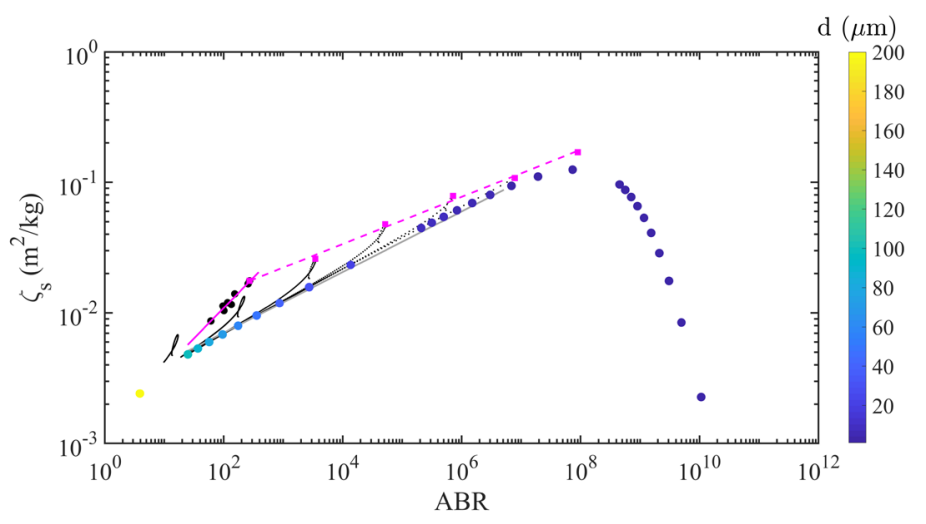

Figure 5. Conversion function of $\zeta_{s}$ as a function of ABR. Magenta solid line: validated domain of ABR by means of acquired samples, magenta dashed line: upper boundary for $\zeta_{s}$, solid grey line: monogranular case and lower boundary for $\zeta_{s}$.

\subsection{Computing the concentration}

To determine the concentration the conversion function derived in the previous section is used to convert the measured $\mathrm{ABR}$ values in $\zeta_{s}$ in order to compute the concentration by means of equation (6). The echo time-series acquired in the year 2016 is used to test the presented method. The results are plotted in Figure 6 considering both the monogranular case (black) and using the obtained conversion functions (magenta). A zoom of this plot for the period $2016 / 05 / 01$ to $2016 / 05 / 31$, indicated by the vertical dashed lines is shown in Figure 7 . The 
computed concentration time-series are relatively close to each other and exhibit a similar behaviour. In this model, they represent the upper concentration limit (obtained with the monogranular model) and lower limits of the concentration.

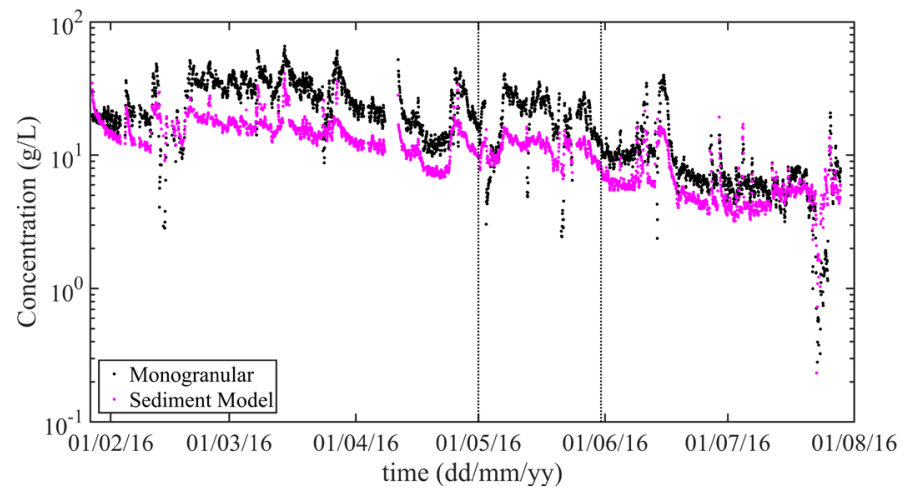

Figure 6. Time-series of the suspended sediment concentration measured in Kokel (black line: monogranular model; magenta: from sediment samples).

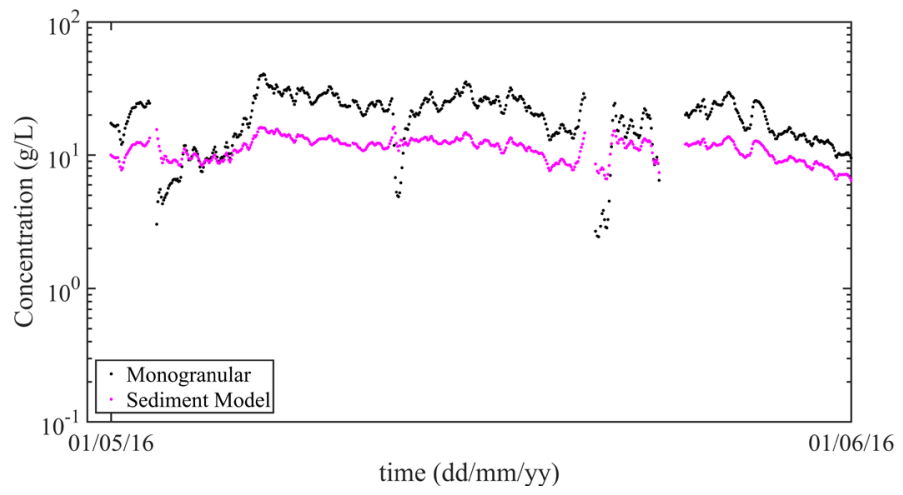

Figure 7. Zoom of the time-series of the suspended sediment concentration measured in Kokel between 2016/05/01 and 2016/05/31.

To define if the obtained conversion functions are close to the monogranular conversion function, the ratio, $R_{C}=\log _{10}\left(C_{\text {mono }} / C_{x}\right)$ is introduced. Where $C_{\text {mono }}$ is the concentration determined using the monogranular conversion function and $C_{x}$ is the concentration determined using the conversion functions derived in section 3.1. It was observed that $-0.5 \leq R_{C} \leq 0.5$, corresponding to a one-order magnitude interval.

Finally, the $2 \mathrm{D}$ probability distribution of the concentration values in function of the ABR is depicted in Figure 8. It is possible to see that there is a negative correlation between the concentration and the $A B R$ values, indicating that low $A B R$ values can result in higher concentrations. 


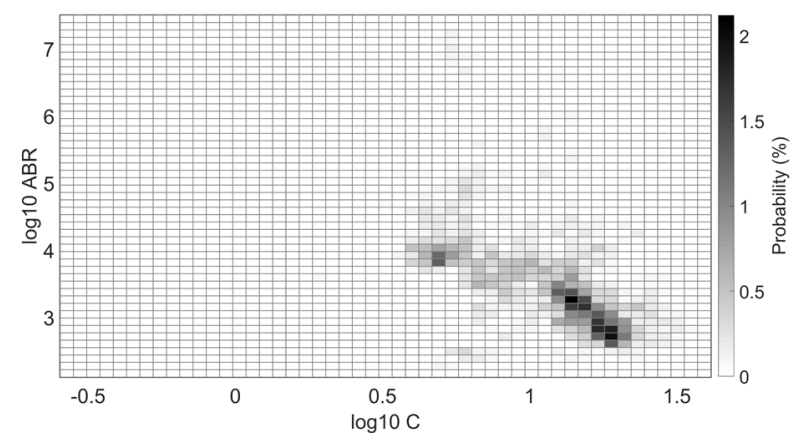

Figure 8. Probability distribution of the concentration in function of the ABR.

\section{Conclusions}

The application of a method to determine the suspended sediment concentration from the echo profiles was used to determine the suspended sediment concentration at the Kokel monitoring station in the Devoll river, Albania. The data was acquired by means of an ADCP working at $0.6 \mathrm{MHz}$ and it was processed by a code developed around the method described in section 1 .

The proposed method relies on the attenuation to backscatter ratio (ABR) and its relationship with the normalised attenuation coefficient to convert the ABR in $\zeta_{s}$. A validation function is required and two options were proposed. One based on the monogranular distribution and another based on the physical properties of sediments sampled from the Devoll river at Kokel. Although these samples did not cover the full ABR range, a conversion function was obtained for remaining range of ABR by considering the limiting upper values of each analytical conversion curve.

To improve this method two actions must be taken: (i) to obtain a validation function for a wider range of $\mathrm{ABR}$ values and (ii) to determine a suitable parameter $C$ valid for long time-series. Finally the computed results should be computed against direct measurements of concentration to determine the method's performance.

Finally, the presented method, despite its limitations is quite general and can be used in other rivers provided that a suitable and validated conversion function of ABR into $\zeta_{s}$ is available.

\section{References}

[1] M. Guerrero, V. Federico, Adv. Water Resour. 113, 167-179 (2018)

[2] M. Guerrero, N. Rüther, R. Szupiany, S. Haun, S. Baranya, F. Latosinski, Water-MDPI 8, 13 (2016)

[3] A.R. Frey, A.B. Coppens, J.V. Sanders, L.E. Kinsler, Fundamentals of Acoustics (Wiley, 1999)

[4] H. Medwin, C. Clay, Fundamentals of Acoustical Oceanography (Academic Press: London, UK, ., 1998)

[5] S. Moore, J.L. Coz, D. Hurther, A. Paquier, Cont. Shelf Res. 46, 50-63 (2012)

[6] P. Thorne, R. Meral, Cont. Shelf Res. 28, 309-317. (2008)

[7] D. Hanes, Cont. Shelf Res. 46, 64-66 (2012) 